\title{
A conservative numerical scheme for Rosenau-RLW equation based on multiple integral finite volume method
}

\author{
Cui Guo ${ }^{1 *}$, Fang Li ${ }^{1}$, Wenping Zhang ${ }^{1}$ and Yuesheng Luo
}

"Correspondence:

2185835@163.com

'Harbin Engineering University,

Harbin, P.R. China

\begin{abstract}
A multiple integral finite volume method combined and Lagrange interpolation are applied in this paper to the Rosenau-RLW (RRLW) equation. We construct a two-level implicit fully discrete scheme for the RRLW equation. The numerical scheme has the accuracy of third order in space and second order in time, respectively. The solvability and uniqueness of the numerical solution are shown. We verify that the numerical scheme keeps the original equation characteristic of energy conservation. It is proved that the numerical scheme is convergent in the order of $O\left(\tau^{2}+h^{3}\right)$ and unconditionally stable. A numerical experiment is given to demonstrate the validity and accuracy of scheme.
\end{abstract}

Keywords: Multiple integral finite volume method; Rosenau-RLW equation; Lagrange interpolation; Brouwer fixed point theorem

\section{Introduction}

It is well known that nonlinear partial differential equations exist in many areas of mathematical physics and fluid mechanics. In the nonlinear evolution equations, the Kortewegde Vries (KdV) and Rosenau-RLW (RLW) equations are two typical cases, given by

$$
u_{t}+u_{x x x}+u u_{x}=0
$$

and

$$
u_{t}-u_{x x t}+u_{x}+u u_{x}=0 .
$$

The KdV equation (1.1) is a nonlinear model used to study the change forms of long waves propagating in a rectangular channel. The RLW equation (1.2) is used to simulate wave motion in media with nonlinear wave steeping and dispersion. The RLW equation was proposed by Peregrine [1, 2] based on the classical KdV equation, and an explanation of different situations of a nonlinear dispersive wave was given in his research.

At the same time, we notice that the motion described by the RLW equation has the same approximate boundary as the $\mathrm{KdV}$ equation. It is well known that the KdV equation has corresponding shortcomings. With the aim to overcome these unavoidable shortcomings

(c) The Author(s) 2019. This article is distributed under the terms of the Creative Commons Attribution 4.0 International License (http://creativecommons.org/licenses/by/4.0/), which permits unrestricted use, distribution, and reproduction in any medium, provided you give appropriate credit to the original author(s) and the source, provide a link to the Creative Commons license, and indicate if changes were made. 
of the KdV equation, Rosenau [3, 4] introduced an equation

$$
u_{t}+u_{x x x x t}+u_{x}+u u_{x}=0
$$

The theoretical studies, on the existence, uniqueness and regularity for the solution of (1.3), have been performed by Park [5]. Various numerical techniques have been used to solve the Rosenau equation [6-12], particularly including the discontinuous Galerkin method, the C1-conforming finite element method [13], the finite difference method and the orthogonal cubic spline collocation method. More detailed solving processes can be obtained in Refs. [14-19].

On the other hand, for further understanding more general nonlinear behaviors of the waves, the term $-u_{x x t}$ needs to be considered. So we address

$$
u_{t}-u_{x x t}+u_{x x x x t}+u_{x}+u u_{x}=0 .
$$

This equation is usually called the Rosenau-RLW (RRLW) equation. Zuo et al. [20] proposed a Crank-Nicolson finite difference method for the RRLW equation. Meanwhile, a three-level difference scheme for (1.4) is investigated by Pan et al. [21]. Furthermore, the finite element approximate solution is used to solve (1.4) and related error estimations for both semi-discrete and fully discrete Galerkin methods are established [22-24]. The coupling equation of KdV and RRLW is also solved through a three-level average implicit finite difference scheme, showing second-order accuracy in space and time, simultaneously [25]. In Ref. [26], the Galerkin cubic B-spline finite element method is proposed to construct the numerical scheme for the RRLW equation. Pan [27] investigated the C-N scheme of RRLW equation through a more classic finite difference approach, and corresponding solvability and convergence have been proved. In addition, the difference scheme for the general RRLW equation is constructed by Wang [28] with some theoretical proofs.

The main contribution of the current work is to present a two-level implicit numerical scheme for the following RRLW equation with some theoretic analysis:

$$
u_{t}-u_{x x t}+u_{x x x x t}+u_{x}+u u_{x}=0, \quad(x, t) \in\left(x_{l}, x_{r}\right) \times[0, T]
$$

with the initial condition

$$
u(x, 0)=u_{0}(x), \quad x \in\left[x_{l}, x_{r}\right]
$$

and boundary conditions

$$
u\left(x_{l}, t\right)=u\left(x_{r}, t\right)=0, \quad u_{x x}\left(x_{l}, t\right)=u_{x x}\left(x_{r}, t\right)=0, \quad t \in[0, T] .
$$

Throughout this paper, we assume that the initial condition $u_{0}(x)$ is sufficiently smooth as required by the error analysis. The system (1.5)-(1.7) is known to satisfy the following conservative law:

$$
E(t)=\|u\|^{2}+\left\|u_{x}\right\|^{2}+\left\|u_{x x}\right\|^{2}=E(0) .
$$


The contents of this paperis as follows. Firstly, in Sect. 2, we present some notations and lemmas. In Sect. 3, we propose a multiple integral finite volume method which is a tool for discrete partial differential equations. Thus a two-level implicit numerical scheme for the RRLW equation is obtained. Next we discuss the discrete energy conservative laws of the numerical scheme and prove its solvability and uniqueness in Sect. 3. We give prior estimates of the numerical scheme in Sect. 4 and prove that the numerical scheme is convergent and stable in Sect. 5. Finally, the error analysis and energy analysis of numerical examples are given in Sect. 6.

In fact, the numerical discrete scheme with parameters constructed by us shows all the discrete schemes that can be constructed by the finite difference method. By choosing the undetermined parameters, we find the best discrete scheme. The best discrete scheme can preserve the conservation property of the original differential equation well. At the same time, for unknown functions in the original differential equation, this method reduces greatly the requirements for the unknown functions in terms of mathematics. More importantly, we explain the concrete and detailed methods about improving the accuracy of the numerical discrete scheme.

\section{Some notations and lemmas}

\subsection{Some notations}

Let $h$ and $\tau$ be the uniform step sizes in the spatial direction and temporal direction, respectively, where $f=\frac{x_{r}-x_{l}}{J}$ and $\tau=\frac{T}{N}$.

Denote $x_{j}=x_{l}+j h(0 \leq j \leq J), t_{n}=n \tau(0 \leq n \leq N)$ and $u_{j}^{n} \approx u\left(x_{l}+j h, n \tau\right)$. Denote the grid $\Omega_{h}=\left\{x_{j} \mid j=0,1, \ldots, J\right\}$ and $Z_{h}^{0}=\left\{u_{j} \mid u_{0}=u_{J}=0, j=0,1, \ldots, J\right\}$. As usual, the following notations will be used:

$$
\begin{aligned}
& \left(u_{j}^{n+\frac{1}{2}}\right)_{\hat{t}}=\frac{u_{j}^{n+1}-u_{j}^{n}}{\tau}, \quad u_{j}^{n+\frac{1}{2}}=\frac{u_{j}^{n+1}+u_{j}^{n}}{2}, \\
& \left(u_{j}^{n}\right)_{x}=\frac{u_{j+1}^{n}-u_{j}^{n}}{h}, \quad\left(u_{j}^{n}\right)_{\bar{x}}=\frac{u_{j}^{n}-u_{j-1}^{n}}{h}, \\
& \left(u_{j}^{n}\right)_{\widehat{x}}=\frac{u_{j+1}^{n}-u_{j-1}^{n}}{2 h}, \quad\left(u_{j}^{n}\right)_{x \bar{x}}=\left(u_{j}^{n}\right)_{\bar{x} x}=\frac{u_{j+1}^{n}-2 u_{j}^{n}+u_{j-1}^{n}}{h^{2}}, \\
& \left(u_{j}^{n+\frac{1}{2}}\right)_{x x \overline{x x}}=\frac{u_{j-2}^{n+\frac{1}{2}}-4 u_{j-1}^{n+\frac{1}{2}}+6 u_{j}^{n+\frac{1}{2}}-4 u_{j+1}^{n+\frac{1}{2}}+u_{j+2}^{n+\frac{1}{2}}}{h^{4}}, \quad\left(u^{n}, v^{n}\right)=h \sum_{j} u_{j}^{n} v_{j}^{n}, \\
& \left\|u^{n}\right\|=\sqrt{\left(u^{n}, u^{n}\right),} \quad\left\|u^{n}\right\|_{\infty}=\max _{0 \leq j \leq J}\left|u_{j}^{n}\right| .
\end{aligned}
$$

In this paper we denote by $C$ a positive constant, which may be of different values on different occasions.

\subsection{Some lemmas}

Lemma 2.1 For any two mesh functions $u, v \in Z_{h}^{0}$, we have

$$
\left(u_{x}, v\right)=-\left(u, v_{\bar{x}}\right), \quad\left(u_{x \bar{x}}, v\right)=-\left(u_{x}, v_{x}\right), \quad\left(u_{x \bar{x}}, u\right)=-\left(u_{x}, u_{x}\right)=-\left\|u_{x}\right\|^{2} .
$$

Furthermore, if $\left(u_{0}^{n}\right)_{x \bar{x}}=\left(u_{J}^{n}\right)_{x \bar{x}}=0$, then $\left(\left(u^{n}\right)_{x x \overline{x x}}, u^{n}\right)=\left\|u_{x x}^{n}\right\|^{2}$. 
Lemma 2.2 $2 B-E$ is a positive definite matrix, where matrix $E$ is the identity matrix of order $J+1$ and

$$
B=\left[\begin{array}{ccccccc}
1 & 0 & 0 & \cdots & 0 & 0 & 0 \\
0 & 7 & 1 & \cdots & 0 & 0 & 0 \\
0 & 1 & 7 & \cdots & 0 & 0 & 0 \\
\vdots & \vdots & \vdots & \ddots & \vdots & \vdots & \vdots \\
0 & 0 & 0 & \cdots & 7 & 1 & 0 \\
0 & 0 & 0 & \cdots & 1 & 7 & 0 \\
0 & 0 & 0 & \cdots & 0 & 0 & 1
\end{array}\right]_{(J+1) \times(J+1)}
$$

Proof We know that

$$
2 B-E=\left[\begin{array}{ccccccc}
1 & 0 & 0 & \cdots & 0 & 0 & 0 \\
0 & 13 & 2 & \cdots & 0 & 0 & 0 \\
0 & 2 & 13 & \cdots & 0 & 0 & 0 \\
\vdots & \vdots & \vdots & \ddots & \vdots & \vdots & \vdots \\
0 & 0 & 0 & \cdots & 13 & 2 & 0 \\
0 & 0 & 0 & \cdots & 2 & 13 & 0 \\
0 & 0 & 0 & \cdots & 0 & 0 & 1
\end{array}\right]_{(J+1) \times(J+1)}
$$

Let $B_{1}=[1], B_{2}=\left[\begin{array}{ll}1 & 0 \\ 0 & 13\end{array}\right], B_{3}=\left[\begin{array}{lll}1 & 0 & 0 \\ 0 & 13 & 2 \\ 0 & 2 & 13\end{array}\right], B_{J+1}=2 B-E$. It is obvious that $\left|B_{1}\right|=1>0,\left|B_{2}\right|=$ $13>0,\left|B_{3}\right|=165>0$ and $\left\{\left|B_{j}\right|\right\}(j=4, \ldots, J)$ obeys

$$
\left|B_{j}\right|=13\left|B_{j-1}\right|-\left|B_{j-2}\right|+\left|B_{j-3}\right| .
$$

We can get

$$
\begin{aligned}
& \left|B_{4}\right|=13\left|B_{3}\right|-\left|B_{2}\right|+\left|B_{1}\right|, \\
& \left|B_{5}\right|=13\left|B_{4}\right|-\left|B_{3}\right|+\left|B_{2}\right|, \\
& \cdots \\
& \left|B_{J}\right|=13\left|B_{J-1}\right|-\left|B_{J-2}\right|+\left|B_{J-3}\right| .
\end{aligned}
$$

We assume that $\left|B_{1}\right|,\left|B_{2}\right|, \ldots,\left|B_{J-1}\right|>0$. We want to prove $\left|B_{J}\right|>0$. From (2.1),

$$
\begin{aligned}
\left|B_{J}\right| & =12\left(\left|B_{J-1}\right|+\cdots+\left|B_{3}\right|\right)-\left|B_{J-2}\right|+\left|B_{3}\right|+\left|B_{1}\right| \\
& =11\left(\left|B_{J-1}\right|+\cdots+\left|B_{3}\right|\right)+\left(\left|B_{J-1}\right|+\left|B_{J-3}\right|+\cdots+\left|B_{3}\right|\right)+166>0 .
\end{aligned}
$$

By the assumption, we have

$$
\left|B_{J+1}\right|=\left|B_{J}\right|>0
$$

So $2 B-E$ is a positive definite matrix. 
Lemma 2.3 (Fixed point theorem [29]) $H$ is a finite dimensional inner product space. We assume $g: H \rightarrow H$ and $g$ is continuous. If there exists $\alpha>0$, for any $x$ in $H$, as long as $\|x\|=$ $\alpha$, we have $(g(x), x)>0$. Then there must exist $x^{*} \in H\left(\left\|x^{*}\right\| \leq \alpha\right)$, which obeys $g\left(x^{*}\right)=0$.

Lemma 2.4 (Discrete Sobolev's inequality [29]) For any discrete function $u_{h}$ and for any given $\varepsilon>0$, there exists a constant $K(\varepsilon, n)$, depending only on $\varepsilon$ and $n$, such that

$$
\|u\|_{\infty} \leq \varepsilon\left\|u_{x}^{n}\right\|+K(\varepsilon, n)\left\|u^{n}\right\| .
$$

Lemma 2.5 (Discrete Gronwall's inequality [30]) Suppose that the discrete function $w_{h}$ satisfies the recurrence formula

$$
w_{n}-w_{n-1} \leq A \tau w_{n}+B \tau w_{n-1}+C_{n} \tau,
$$

where $A, B, C_{n}(n=1,2, \ldots, N)$ are nonnegative constants. Then

$$
\left\|w_{n}\right\|_{\infty} \leq\left(w_{0}+\tau \sum_{k=1}^{N} C_{k}\right) e^{2(A+B) \tau},
$$

where $\tau$ is small, such that $(A+B) \tau \leq \frac{N-1}{2 N}(N>1)$.

\section{An implicit conservative numerical scheme and its discrete conservative law}

\subsection{The multiple integral finite volume method}

It is necessary to introduce the multiple integral finite volume method (MIFVM) briefly. The method is a new approximation method for solving partial differential equation, which is proposed by Yuesheng Luo. The basic idea is to make the original partial differential equation to be an integral equation by a certain number of integrations in the spatial $x$ direction. The goal is that the integral equation no longer contains the derivative item of the unknown function. In this way, we avoid handling the approximation as regards the derivative term.

Firstly, the number of integrations depends on the order of the highest derivative in the spatial $x$ direction of the unknown function in the partial differential equation. The relationship between $m$ and $r$ satisfies $m=2^{r}-1$, where $m$ is the number of integrations and $r$ is the order of the highest derivative of the unknown function in the spatial $x$ direction.

Secondly, is it well known that the original partial differential equation usually is expressed by the derivative of an unknown function and an unknown function, for example $u_{x}, u_{x x}, u_{x x x}, u_{x x x x}$ and $u$. The MIFVM is to transform the original partial differential equation into an integral equation, which is expressed by $u_{j}^{n} \cdot u_{j}^{n} \approx u\left(x_{l}+j h, n \tau\right)$ is the unknown function's approximate value at the grid node. If there is $u_{j+\varepsilon}^{n}$, which is not at the grid node, in the integral equation, we often use the Lagrange polynomial to deal with it. This step is just an approximation to the original function.

For example, for (1.5), the order of the highest derivative of unknown function is four. By $m=2^{4}-1$, we should make 15 times integrations for every item, as shown by

$$
\int_{x x x x} u_{t}-\int_{x x x x} u_{x x t}+\int_{x x x x} u_{x x x x t}+\int_{x x x x} u_{x}+\int_{x x x x} u u_{x}=0,
$$


where

$$
\begin{aligned}
\int_{x x x x} u \stackrel{\operatorname{def}}{=} & \int_{x_{j}+\varepsilon_{7}}^{x_{j}+\varepsilon_{8}} d x_{f_{2}} \int_{x_{j}+\varepsilon_{6}}^{x_{j}+\varepsilon_{7}} d x_{f_{1}} \int_{x_{j}+\varepsilon_{5}}^{x_{j}+\varepsilon_{6}} d x_{e_{2}} \int_{x_{j}}^{x_{j}+\varepsilon_{5}} d x_{e_{1}} \\
& \times \int_{x_{j}-\varepsilon_{4}}^{x_{j}} d x_{d_{2}} \int_{x_{j}-\varepsilon_{3}}^{x_{j}-\varepsilon_{4}} d x_{d_{1}} \int_{x_{j}-\varepsilon_{2}}^{x_{j}-\varepsilon_{3}} d x_{c_{2}} \\
& \times \int_{x_{j}-\varepsilon_{1}}^{x_{j}-\varepsilon_{2}} d x_{c_{1}} \int_{x_{f_{1}}}^{x_{f_{2}}} d x_{f} \int_{x_{e_{1}}}^{x_{e_{2}}} d x_{e} \int_{x_{d_{1}}}^{x_{d_{2}}} d x_{d} \int_{x_{c_{1}}}^{x_{c_{2}}} d x_{c} \\
& \times \int_{x_{e}}^{x_{f}} d x_{b} \int_{x_{c}}^{x_{d}} d x_{a} \int_{x_{a}}^{x_{b}} u d x,
\end{aligned}
$$

$\varepsilon_{i} \in R, i=1,2, \ldots, 8$. The center difference is used to deal with the first derivative in the time direction. Then we can get a numerical scheme for the original equation.

\subsection{A two-level implicit numerical scheme}

In order to get a numerical scheme, which can preserve some properties of the original equation, applying MIFVM to Eq. (1.5) on time $n+\frac{1}{2}$ level, we let $\varepsilon_{1}=-\varepsilon_{4}=-\varepsilon_{5}=\varepsilon_{8}=$ $\sqrt{3} h, \varepsilon_{2}=-\varepsilon_{3}=-\varepsilon_{6}=\varepsilon_{7}=\frac{\sqrt{3}}{3} h$. So we can get

$$
\begin{aligned}
& \int_{x_{j}+\frac{\sqrt{3}}{3} h}^{x_{j}+\sqrt{3} h} d x_{f_{2}} \int_{x_{j}-\frac{\sqrt{3}}{3} h}^{x_{j}+\frac{\sqrt{3}}{3} h} d x_{f_{1}} \int_{x_{j}-\sqrt{3} h}^{x_{j}-\frac{\sqrt{3}}{3} h} d x_{e_{2}} \int_{x_{j}-\sqrt{3} h}^{x_{j}} d x_{e_{1}} \int_{x_{j}}^{x_{j}+\sqrt{3} h} d x_{d_{2}} \\
& \times \int_{x_{j}+\frac{\sqrt{3}}{3} h}^{x_{j}+\sqrt{3} h} d x_{d_{1}} \int_{x_{j}-\frac{\sqrt{3}}{3} h}^{x_{j}+\frac{\sqrt{3}}{3} h} d x_{c_{2}} \int_{x_{j}-\sqrt{3} h}^{x_{j}-\frac{\sqrt{3}}{3} h} d x_{c_{1}} \\
& \times \int_{x_{f_{1}}}^{x_{f_{2}}} d x_{f} \int_{x_{e_{1}}}^{x_{e_{2}}} d x_{e} \int_{x_{d_{1}}}^{x_{d_{2}}} d x_{d} \int_{x_{c_{1}}}^{x_{c_{2}}} d x_{c} \int_{x_{e}}^{x_{f}} d x_{b} \int_{x_{c}}^{x_{d}} d x_{a} \int_{x_{a}}^{x_{b}} u_{t} d x \\
& -\int_{x_{j}+\frac{\sqrt{3}}{3} h}^{x_{j}+\sqrt{3} h} d x_{f_{2}} \int_{x_{j}-\frac{\sqrt{3}}{3} h}^{x_{j}+\frac{\sqrt{3}}{3} h} d x_{f_{1}} \int_{x_{j}-\sqrt{3} h}^{x_{j}-\frac{\sqrt{3}}{3} h} d x_{e_{2}} \int_{x_{j}-\sqrt{3} h}^{x_{j}} d x_{e_{1}} \int_{x_{j}}^{x_{j}+\sqrt{3} h} d x_{d_{2}} \\
& \times \int_{x_{j}+\frac{\sqrt{3}}{3} h}^{x_{j}+\sqrt{3} h} d x_{d_{1}} \int_{x_{j}-\frac{\sqrt{3}}{3} h}^{x_{j}+\frac{\sqrt{3}}{3} h} d x_{c_{2}} \int_{x_{j}-\sqrt{3} h}^{x_{j}-\frac{\sqrt{3}}{3} h} d x_{c_{1}} \\
& \times \int_{x_{f_{1}}}^{x_{f_{2}}} d x_{f} \int_{x_{e_{1}}}^{x_{e_{2}}} d x_{e} \int_{x_{d_{1}}}^{x_{d_{2}}} d x_{d} \int_{x_{c_{1}}}^{x_{c_{2}}} d x_{c} \int_{x_{e}}^{x_{f}} d x_{b} \int_{x_{c}}^{x_{d}} d x_{a} \int_{x_{a}}^{x_{b}} u_{x x t} d x \\
& +\int_{x_{j}+\frac{\sqrt{3}}{3} h}^{x_{j}+\sqrt{3} h} d x_{f_{2}} \int_{x_{j}-\frac{\sqrt{3}}{3} h}^{x_{j}+\frac{\sqrt{3}}{3} h} d x_{f_{1}} \int_{x_{j}-\sqrt{3} h}^{x_{j}-\frac{\sqrt{3}}{3} h} d x_{e_{2}} \int_{x_{j}-\sqrt{3} h}^{x_{j}} d x_{e_{1}} \int_{x_{j}}^{x_{j}+\sqrt{3} h} d x_{d_{2}} \\
& \times \int_{x_{j}+\frac{\sqrt{3}}{3} h}^{x_{j}+\sqrt{3} h} d x_{d_{1}} \int_{x_{j}-\frac{\sqrt{3}}{3} h}^{x_{j}+\frac{\sqrt{3}}{3} h} d x_{c_{2}} \int_{x_{j}-\sqrt{3} h}^{x_{j}-\frac{\sqrt{3}}{3} h} d x_{c_{1}} \\
& \times \int_{x_{f_{1}}}^{x_{f_{2}}} d x_{f} \int_{x_{e_{1}}}^{x_{e_{2}}} d x_{e} \int_{x_{d_{1}}}^{x_{d_{2}}} d x_{d} \int_{x_{c_{1}}}^{x_{c_{2}}} d x_{c} \int_{x_{e}}^{x_{f}} d x_{b} \int_{x_{c}}^{x_{d}} d x_{a} \int_{x_{a}}^{x_{b}} u_{x x x x t} d x \\
& +\int_{x_{j}+\frac{\sqrt{3}}{3} h}^{x_{j}+\sqrt{3} h} d x_{f_{2}} \int_{x_{j}-\frac{\sqrt{3}}{3} h}^{x_{j}+\frac{\sqrt{3}}{3} h} d x_{f_{1}} \int_{x_{j}-\sqrt{3} h}^{x_{j}-\frac{\sqrt{3}}{3} h} d x_{e_{2}} \int_{x_{j}-\sqrt{3} h}^{x_{j}} d x_{e_{1}} \int_{x_{j}}^{x_{j}+\sqrt{3} h} d x_{d_{2}} \\
& \times \int_{x_{j}+\frac{\sqrt{3}}{3} h}^{x_{j}+\sqrt{3} h} d x_{d_{1}} \int_{x_{j}-\frac{\sqrt{3}}{3} h}^{x_{j}+\frac{\sqrt{3}}{3} h} d x_{c_{2}} \int_{x_{j}-\sqrt{3} h}^{x_{j}-\frac{\sqrt{3}}{3} h} d x_{c_{1}}
\end{aligned}
$$




$$
\begin{aligned}
& \times \int_{x_{f_{1}}}^{x_{f_{2}}} d x_{f} \int_{x_{e_{1}}}^{x_{e_{2}}} d x_{e} \int_{x_{d_{1}}}^{x_{d_{2}}} d x_{d} \int_{x_{c_{1}}}^{x_{c_{2}}} d x_{c} \int_{x_{e}}^{x_{f}} d x_{b} \int_{x_{c}}^{x_{d}} d x_{a} \int_{x_{a}}^{x_{b}} u_{x} d x \\
& +\int_{x_{j}+\frac{\sqrt{3}}{3} h}^{x_{j}+\sqrt{3} h} d x_{f_{2}} \int_{x_{j}-\frac{\sqrt{3}}{3} h}^{x_{j}+\frac{\sqrt{3}}{3} h} d x_{f_{1}} \int_{x_{j}-\sqrt{3} h}^{x_{j}-\frac{\sqrt{3}}{3} h} d x_{e_{2}} \int_{x_{j}-\sqrt{3} h}^{x_{j}} d x_{e_{1}} \int_{x_{j}}^{x_{j}+\sqrt{3} h} d x_{d_{2}} \\
& \times \int_{x_{j}+\frac{\sqrt{3}}{3} h}^{x_{j}+\sqrt{3} h} d x_{d_{1}} \int_{x_{j}-\frac{\sqrt{3}}{3} h}^{x_{j}+\frac{\sqrt{3}}{3} h} d x_{c_{2}} \int_{x_{j}-\sqrt{3} h}^{x_{j}-\frac{\sqrt{3}}{3} h} d x_{c_{1}} \\
& \times \int_{x_{f_{1}}}^{x_{f_{2}}} d x_{f} \int_{x_{e_{1}}}^{x_{e_{2}}} d x_{e} \int_{x_{d_{1}}}^{x_{d_{2}}} d x_{d} \int_{x_{c_{1}}}^{x_{c_{2}}} d x_{c} \int_{x_{e}}^{x_{f}} d x_{b} \int_{x_{c}}^{x_{d}} d x_{a} \int_{x_{a}}^{x_{b}} u u_{x} d x=0 .
\end{aligned}
$$

There are five integration items. For the first item, the approximation of the first-order derivative in the time direction is

$$
u_{t}\left(x, t^{n+\frac{1}{2}}\right)=\frac{u^{n+1}(x)-u^{n}(x)}{\tau}+O\left(\tau^{2}\right) .
$$

For the third item, we use the five points Lagrange interpolation for the $x$ direction. It is

$$
\begin{aligned}
u(x, t)= & \frac{\left(x-x_{j-1}\right)\left(x-x_{j}\right)\left(x-x_{j+1}\right)\left(x-x_{j+2}\right)}{\left(x_{j-2}-x_{j-1}\right)\left(x_{j-2}-x_{j}\right)\left(x_{j-2}-x_{j+1}\right)\left(x_{j-2}-x_{j+2}\right)} u_{j-2}(t) \\
& +\frac{\left(x-x_{j-2}\right)\left(x-x_{j}\right)\left(x-x_{j+1}\right)\left(x-x_{j+2}\right)}{\left(x_{j-1}-x_{j-2}\right)\left(x_{j-1}-x_{j}\right)\left(x_{j-1}-x_{j+1}\right)\left(x_{j-1}-x_{j+2}\right)} u_{j-1}(t) \\
& +\frac{\left(x-x_{j-2}\right)\left(x-x_{j-1}\right)\left(x-x_{j+1}\right)\left(x-x_{j+2}\right)}{\left(x_{j}-x_{j-2}\right)\left(x_{j}-x_{j-1}\right)\left(x_{j}-x_{j+1}\right)\left(x_{j}-x_{j+2}\right)} u_{j}(t) \\
& +\frac{\left(x-x_{j-2}\right)\left(x-x_{j-1}\right)\left(x-x_{j}\right)\left(x-x_{j+2}\right)}{\left(x_{j+1}-x_{j-2}\right)\left(x_{j+1}-x_{j-1}\right)\left(x_{j+1}-x_{j}\right)\left(x_{j+1}-x_{j+2}\right)} u_{j+1}(t) \\
& +\frac{\left(x-x_{j-2}\right)\left(x-x_{j-1}\right)\left(x-x_{j}\right)\left(x-x_{j+1}\right)}{\left(x_{j+2}-x_{j-2}\right)\left(x_{j+2}-x_{j-1}\right)\left(x_{j+2}-x_{j}\right)\left(x_{j+2}-x_{j+1}\right)} u_{j+2}(t)+O\left(h^{5}\right) .
\end{aligned}
$$

For the other three items, we use three points Lagrange interpolation for the $x$ direction. It is

$$
\begin{aligned}
u(x, t)= & \frac{\left(x-x_{j}\right)\left(x-x_{j+1}\right)}{\left(x_{j-1}-x_{j}\right)\left(x_{j-1}-x_{j+1}\right)} u_{j-1}(t)+\frac{\left(x-x_{j-1}\right)\left(x-x_{j+1}\right)}{\left(x_{j}-x_{j-1}\right)\left(x_{j}-x_{j+1}\right)} u_{j}(t) \\
& +\frac{\left(x-x_{j-1}\right)\left(x-x_{j}\right)}{\left(x_{j+1}-x_{j-1}\right)\left(x_{j+1}-x_{j}\right)} u_{j+1}(t)+O\left(h^{3}\right) .
\end{aligned}
$$

Substituting (3.4), (3.5) and (3.6) into (3.3) and simplifying, we get a two-level implicit scheme for equation (1.5). We obtain

$$
\begin{aligned}
& \frac{1}{9}\left(u_{j-1}^{n+\frac{1}{2}}+7 u_{j}^{n+\frac{1}{2}}+u_{j+1}^{n+\frac{1}{2}}\right)_{\widehat{t}}-\left(u_{j}^{n+\frac{1}{2}}\right)_{x \widehat{x} \hat{t}}+\left(u_{j}^{n+\frac{1}{2}}\right)_{x x \overline{x x} \hat{t}}+\left(u_{j}^{n+\frac{1}{2}}\right)_{\widehat{x}} \\
& \quad+\frac{1}{3}\left(u_{j}^{n+\frac{1}{2}}\right)_{\widehat{x}}\left(u_{j-1}^{n+\frac{1}{2}}+u_{j}^{n+\frac{1}{2}}+u_{j+1}^{n+\frac{1}{2}}\right)=0, \quad 1 \leq j \leq J-1,1 \leq n \leq N-1, \\
& u_{j}^{0}=u_{0}\left(x_{j}\right), \quad 1 \leq j \leq J-1, \\
& u_{0}^{n}=u_{J}^{n}=0, \quad\left(u_{0}^{n}\right)_{x \bar{x}}=\left(u_{J}^{n}\right)_{x \bar{x}}=0, \quad 1 \leq n \leq N-1 .
\end{aligned}
$$




\subsection{Conservative law of the discrete format}

Theorem 3.1 The two-level implicit numerical scheme (3.7) admits the following invariant:

$$
E^{n}=\frac{7}{9}\left\|u^{n}\right\|^{2}+\frac{2 h}{9} \sum_{j=1}^{J-1} u_{j}^{n} u_{j+1}^{n}+\left\|u_{x}^{n}\right\|^{2}+\left\|u_{x x}^{n}\right\|^{2}=E^{n-1}=\cdots=E^{0} .
$$

Proof Computing the inner product of (3.7) with $2 u^{n+\frac{1}{2}}$ (i.e. $u^{n+1}+u^{n}$ ), according to Lemma 2.1, we have

$$
\begin{aligned}
& \frac{7}{9 \tau}\left\|u^{n+1}\right\|^{2}+\frac{2 h}{9 \tau} \sum_{j=1}^{J-1} u_{j}^{n+1} u_{j+1}^{n+1}-\frac{7}{9 \tau}\left\|u^{n}\right\|^{2}+\frac{2 h}{9 \tau} \sum_{j=1}^{J-1} u_{j}^{n} u_{j+1}^{n}+\frac{1}{\tau}\left(\left\|u_{x}^{n+1}\right\|^{2}-\left\|u_{x}^{n}\right\|^{2}\right) \\
& +\frac{1}{\tau}\left(\left\|u_{x x}^{n+1}\right\|^{2}-\left\|u_{x x}^{n}\right\|^{2}\right)+\left(P, 2 u^{n+\frac{1}{2}}\right)=0,
\end{aligned}
$$

where $P=\frac{1}{3}\left(u_{j}^{n+\frac{1}{2}}\right)_{\widehat{x}}\left(u_{j-1}^{n+\frac{1}{2}}+u_{j}^{n+\frac{1}{2}}+u_{j+1}^{n+\frac{1}{2}}\right)$. Now, computing the last term of the left-hand side in (3.10), we get

$$
\begin{aligned}
(P, & \left.2 u^{n+\frac{1}{2}}\right) \\
= & \left(\frac{2}{3}\left(u_{j}^{n+\frac{1}{2}}\right)_{\widehat{x}}\left(u_{j-1}^{n+\frac{1}{2}}+u_{j}^{n+\frac{1}{2}}+u_{j+1}^{n+\frac{1}{2}}\right), u^{n+\frac{1}{2}}\right) \\
= & \frac{1}{3} \sum_{j=1}^{J-1}\left(u_{j+1}^{n+\frac{1}{2}} u_{j}^{n+\frac{1}{2}} u_{j}^{n+\frac{1}{2}}+u_{j+1}^{n+\frac{1}{2}} u_{j}^{n+\frac{1}{2}} u_{j+1}^{n+\frac{1}{2}}-u_{j-1}^{n+\frac{1}{2}} u_{j}^{n+\frac{1}{2}} u_{j-1}^{n+\frac{1}{2}}-u_{j-1}^{n+\frac{1}{2}} u_{j}^{n+\frac{1}{2}} u_{j}^{n+\frac{1}{2}}\right) \\
= & \frac{1}{3}\left[\sum_{j=1}^{J-2}\left(u_{j}^{n+\frac{1}{2}} u_{j}^{n+\frac{1}{2}} u_{j+1}^{n+\frac{1}{2}}+u_{j}^{n+\frac{1}{2}} u_{j+1}^{n+\frac{1}{2}} u_{j+1}^{n+\frac{1}{2}}-u_{j}^{n+\frac{1}{2}} u_{j}^{n+\frac{1}{2}} u_{j+1}^{n+\frac{1}{2}}-u_{j}^{n+\frac{1}{2}} u_{j+1}^{n+\frac{1}{2}} u_{j+1}^{n+\frac{1}{2}}\right)\right] \\
& +u_{J-1}^{n+\frac{1}{2}} u_{J-1}^{n+\frac{1}{2}} u_{J}^{n+\frac{1}{2}}+u_{J-1}^{n+\frac{1}{2}} u_{J}^{n+\frac{1}{2}} u_{J}^{n+\frac{1}{2}} \\
& -\left(u_{0}^{n+\frac{1}{2}} u_{0}^{n+\frac{1}{2}} u_{1}^{n+\frac{1}{2}}+u_{0}^{n+\frac{1}{2}} u_{1}^{n+\frac{1}{2}} u_{1}^{n+\frac{1}{2}}\right)=0 .
\end{aligned}
$$

Substituting (3.11) into (3.10), we have

$$
\begin{aligned}
& \frac{7}{9}\left\|u^{n+1}\right\|^{2}+\frac{2 h}{9} \sum_{j=1}^{J-1} u_{j}^{n+1} u_{j+1}^{n+1}-\frac{7}{9}\left\|u^{n}\right\|^{2} \\
& \quad+\frac{2 h}{9} \sum_{j=1}^{J-1} u_{j}^{n} u_{j+1}^{n}+\left\|u_{x}^{n+1}\right\|^{2}-\left\|u_{x}^{n}\right\|^{2}+\left\|u_{x x}^{n+1}\right\|^{2}-\left\|u_{x x}^{n}\right\|^{2}=0 .
\end{aligned}
$$

We let

$$
E^{n}=\frac{7}{9}\left\|u^{n}\right\|^{2}+\frac{2 h}{9} \sum_{j=1}^{J-1} u_{j}^{n} u_{j+1}^{n}+\left\|u_{x}^{n}\right\|^{2}+\left\|u_{x x}^{n}\right\|^{2}
$$


Then we obtain

$$
E^{n}=\frac{7}{9}\left\|u^{n}\right\|^{2}+\frac{2 h}{9} \sum_{j=1}^{J-1} u_{j}^{n} u_{j+1}^{n}+\left\|u_{x}^{n}\right\|^{2}+\left\|u_{x x}^{n}\right\|^{2}=E^{n-1}=\cdots=E^{0}
$$

The proof is completed.

\subsection{Solvability}

Next, we shall prove the solvability of the difference scheme (3.7).

Theorem 3.2 The finite difference scheme (3.7) is solvable.

Proof For the difference scheme (3.7)-(3.9), we assume that $u^{0}, u^{1}, \ldots, u^{n}(n \leq N-1)$ obey (3.7). Next, we will prove that $u^{n+1}$ also satisfies (3.7). The operation $g$ is defined as follows:

$$
g(v)=\frac{2}{9} A\left(v-u^{n}\right)-2\left(v_{x \bar{x}}-u_{x \bar{x}}^{n}\right)+2 v_{x x \overline{x x}}-2 u_{x x \overline{x x}}^{n}+\tau v_{\widehat{x}}+\frac{\tau}{3}\left(v_{j-1}+v_{j}+v_{j+1}\right) v_{\widehat{x}}
$$

It is obvious that $g$ is continuous. Computing the inner product of (3.13) with $v$, according to Lemma 2.1, we have

$$
\begin{aligned}
(g(v), v)= & \frac{2}{9}(A v, v)-\frac{2}{9}\left(A u^{n}, v\right)-2\left(v_{x \bar{x}}, v\right)+2\left(u_{x \bar{x}}^{n}, v\right)+\left(2 v_{x x \overline{x x}}, v\right)-\left(2 u_{x x \bar{x} x}^{n}, v\right) \\
\geq & \frac{2}{9} v^{T} W^{T} v+\frac{2}{9} v^{T} L^{T} v-\frac{2}{9}\left\|A u^{n}\right\|\|v\|+2\left\|v_{x}\right\|^{2} \\
& -2\left\|u_{x}^{n}\right\|\left\|v_{x}\right\|+2\left\|v_{x x}\right\|^{2}-2\left\|u_{x x}^{n}\right\|\left\|v_{x x}\right\| \\
\geq & \frac{2}{9}\left(\lambda_{0} v_{0}^{2}+\lambda_{2} v_{1}^{2}+\cdots+\lambda_{J} v_{J}^{2}\right)-\frac{1}{9}\left\|A u^{n}\right\|^{2} \\
& -\frac{1}{9}\|v\|^{2}+\left\|v_{x}\right\|^{2}-\left\|u_{x}^{n}\right\|^{2}+\left\|v_{x x}\right\|^{2}-\left\|u_{x x}^{n}\right\|^{2} \\
\geq & \frac{1}{9}\left(2 \lambda_{\min }-1\right)\|v\|^{2}-\frac{1}{9}\left\|A u^{n}\right\|^{2}-\left\|u_{x}^{n}\right\|^{2}-\left\|u_{x x}^{n}\right\|^{2},
\end{aligned}
$$

where

$$
A=\left[\begin{array}{ccccccc}
1 & 0 & 0 & \cdots & 0 & 0 & 0 \\
1 & 7 & 1 & \cdots & 0 & 0 & 0 \\
0 & 1 & 7 & \cdots & 0 & 0 & 0 \\
\vdots & \vdots & \vdots & \ddots & \vdots & \vdots & \vdots \\
0 & 0 & 0 & \cdots & 7 & 1 & 0 \\
0 & 0 & 0 & \cdots & 1 & 7 & 1 \\
0 & 0 & 0 & \cdots & 0 & 0 & 1
\end{array}\right]_{(J+1) \times(J+1)}=W+L
$$




$$
\begin{aligned}
W & =\left[\begin{array}{ccccccc}
1 & 0 & 0 & \cdots & 0 & 0 & 0 \\
0 & 7 & 1 & \cdots & 0 & 0 & 0 \\
0 & 1 & 7 & \cdots & 0 & 0 & 0 \\
\vdots & \vdots & \vdots & \ddots & \vdots & \vdots & \vdots \\
0 & 0 & 0 & \cdots & 7 & 1 & 0 \\
0 & 0 & 0 & \cdots & 1 & 7 & 0 \\
0 & 0 & 0 & \cdots & 0 & 0 & 1
\end{array}\right]_{(J+1) \times(J+1)} \\
L & =\left[\begin{array}{ccccccc}
0 & 0 & 0 & \cdots & 0 & 0 & 0 \\
1 & 0 & 0 & \cdots & 0 & 0 & 0 \\
0 & 0 & 0 & \cdots & 0 & 0 & 0 \\
\vdots & \vdots & \vdots & \ddots & \vdots & \vdots & \vdots \\
0 & 0 & 0 & \cdots & 0 & 0 & 0 \\
0 & 0 & 0 & \cdots & 0 & 0 & 1 \\
0 & 0 & 0 & \cdots & 0 & 0 & 0
\end{array}\right]_{(J+1) \times(J+1)}
\end{aligned}
$$

$\lambda_{\min }$ is the minimum eigenvalue of $W$. By Lemma 2.2 , we know that $2 \lambda_{\min }-1>0$. Let

$$
\|v\|^{2}=\frac{1}{2 \lambda_{\min }-1}\left(\left\|A u^{n}\right\|^{2}+9\left\|u_{x}^{n}\right\|^{2}+9\left\|u_{x x}^{n}\right\|^{2}+1\right) .
$$

We have $(g(v), v)>0, \forall v \in Z_{h}^{0}$. According to Lemma 2.3, there is $v^{*} \in Z_{h}^{0}$ which obeys $g\left(v^{*}\right)=0$. So we let $v^{*}=\frac{u^{n+1}+u^{n}}{2}$, then $u^{n+1}$ obeys (3.7).

Theorem 3.3 The finite difference scheme (3.7) has a unique solution.

Proof Let $I^{n+1}=U^{n+1}-V^{n+1}$, where $U^{n+1}$ and $V^{n+1}$ are both the solution of scheme (3.7). We want to prove that $I^{n+1}=0$. According to (3.7), we get

$$
\begin{aligned}
& \frac{1}{9}\left(\left(I_{j-1}^{n+\frac{1}{2}}\right)_{\widehat{t}}+7\left(I_{j}^{n+\frac{1}{2}}\right)_{\widehat{t}}+\left(I_{j+1}^{n+\frac{1}{2}}\right)_{\widehat{t}}\right)-\left(I_{j}^{n+\frac{1}{2}}\right)_{x \widehat{x} \widehat{t}}+\left(I_{j}^{n+\frac{1}{2}}\right)_{x x \overline{x x \widehat{x}}}+\left(I_{j}^{n+\frac{1}{2}}\right)_{\widehat{x}} \\
& \quad+\varphi\left(u_{j}^{n+\frac{1}{2}}, u_{j}^{n+\frac{1}{2}}\right)-\varphi\left(v_{j}^{n+\frac{1}{2}}, v_{j}^{n+\frac{1}{2}}\right)=0,
\end{aligned}
$$

where $\varphi\left(u_{j}^{n+\frac{1}{2}}, u_{j}^{n+\frac{1}{2}}\right)=\frac{1}{3}\left(u_{j}^{n+\frac{1}{2}}\right)_{\widehat{x}}\left(u_{j-1}^{n+\frac{1}{2}}+u_{j}^{n+\frac{1}{2}}+u_{j+1}^{n+\frac{1}{2}}\right)$. Computing the inner product of (3.12) with $2 I^{n+\frac{1}{2}}\left(I^{n}+I^{n+1}\right)$, we have

$$
\begin{aligned}
& \frac{7}{9 \tau}\left\|I^{n+1}\right\|^{2}+\frac{2 h}{9 \tau} \sum_{j=1}^{J-1} I_{j}^{n+1} I_{j+1}^{n+1}-\frac{7}{9 \tau}\left\|I^{n}\right\|-\frac{2 h}{9 \tau} \sum_{j=1}^{J-1} I_{j}^{n} I_{j+1}^{n} \\
& \quad+\frac{1}{\tau}\left(\left\|I_{x}^{n+1}\right\|^{2}-\left\|I_{x}^{n}\right\|^{2}\right)+\frac{1}{\tau}\left(\left\|I_{x x}^{n+1}\right\|^{2}-\left\|I_{x x}^{n}\right\|^{2}\right) \\
& \quad+\left(\varphi\left(u_{j}^{n+\frac{1}{2}}, u_{j}^{n+\frac{1}{2}}\right)-\varphi\left(v_{j}^{n+\frac{1}{2}}, v_{j}^{n+\frac{1}{2}}\right), I^{n+\frac{1}{2}}\right)=0 .
\end{aligned}
$$

Due to the result that

$$
-\left(\varphi\left(u_{j}^{n+\frac{1}{2}}, u_{j}^{n+\frac{1}{2}}\right)-\varphi\left(v_{j}^{n+\frac{1}{2}}, v_{j}^{n+\frac{1}{2}}\right), I^{n+\frac{1}{2}}\right)
$$




$$
\begin{aligned}
= & -\frac{2}{3} h \sum_{j=1}^{J-1}\left(I_{j-1}^{n+\frac{1}{2}}+I_{j}^{n+\frac{1}{2}}+I_{j+1}^{n+\frac{1}{2}}\right)\left(u_{j}^{n+\frac{1}{2}}\right)_{\widehat{x}} I_{j}^{n+\frac{1}{2}} \\
& -\frac{2}{3} h \sum_{j=1}^{J-1}\left(I_{j-1}^{n+\frac{1}{2}}+I_{j}^{n+\frac{1}{2}}+I_{j+1}^{n+\frac{1}{2}}\right)\left(v_{j}^{n+\frac{1}{2}}\right)_{\widehat{x} j} I_{j}^{n+\frac{1}{2}} \\
& -\frac{2}{3} h \sum_{j=1}^{J-1}\left(v_{j-1}^{n+\frac{1}{2}}+v_{j}^{n+\frac{1}{2}}+v_{j+1}^{n+\frac{1}{2}}\right)\left(u_{j}^{n+\frac{1}{2}}\right)_{\widehat{x}} J_{j}^{n+\frac{1}{2}} \\
& +\frac{2}{3} h \sum_{j=1}^{J-1}\left(u_{j-1}^{n+\frac{1}{2}}+u_{j}^{n+\frac{1}{2}}+u_{j+1}^{n+\frac{1}{2}}\right)\left(v_{j}^{n+\frac{1}{2}}\right)_{\widehat{x} j} I_{j}^{n+\frac{1}{2}} \\
\leq & \frac{2}{3} C h \sum_{j=1}^{J-1}\left(\left|I_{j-1}^{n+\frac{1}{2}}\right|+\left|I_{j}^{n+\frac{1}{2}}\right|+\left|I_{j+1}^{n+\frac{1}{2}}\right|\right)\left|I_{j}^{n+\frac{1}{2}}\right|+\frac{2}{3} C h \sum_{j=1}^{J-1}\left|\left(I_{j}^{n+\frac{1}{2}}\right)_{\widehat{x}}\right|\left|I_{j}^{n+\frac{1}{2}}\right| \\
\leq & C\left(\left\|I^{n+1}\right\|^{2}+\left\|I^{n}\right\|^{2}+\left\|I_{x}^{n+1}\right\|^{2}+\left\|I_{x}^{n}\right\|^{2}\right),
\end{aligned}
$$

we have

$$
\begin{aligned}
\frac{7}{9 \tau}\left\|I^{n+1}\right\|^{2}+\frac{2 h}{9 \tau} \sum_{j=1}^{J-1} I_{j}^{n+1} I_{j+1}^{n+1}-\frac{7}{9 \tau}\left\|I^{n}\right\|^{2}-\frac{2 h}{9 \tau} \sum_{j=1}^{J-1} I_{j}^{n} I_{j+1}^{n} \\
+\frac{1}{\tau}\left(\left\|I_{x}^{n+1}\right\|^{2}-\left\|I_{x}^{n}\right\|^{2}\right)+\frac{1}{\tau}\left(\left\|I_{x x}^{n+1}\right\|^{2}-\left\|I_{x x}^{n}\right\|^{2}\right) \\
\leq C\left(\left\|I^{n+1}\right\|^{2}+\left\|I^{n}\right\|^{2}+\left\|I_{x}^{n+1}\right\|^{2}+\left\|I_{x}^{n}\right\|^{2}\right) .
\end{aligned}
$$

Let

$$
Q^{n}=\frac{7}{9}\left\|I^{n}\right\|^{2}+\frac{2 h}{9} \sum_{j=1}^{J-1} I_{j}^{n} I_{j+1}^{n}+\left\|I_{x}^{n}\right\|^{2}+\left\|I_{x x}^{n}\right\|^{2} .
$$

So we get

$$
Q^{n+1}-Q^{n} \leq C \tau\left(Q^{n+1}+Q^{n}\right)
$$

That is,

$$
Q^{n+1} \leq \frac{1+C \tau}{1-C \tau} Q^{n}=(1+\vartheta \tau) Q^{n},
$$

where $\vartheta=\frac{2 C}{1-C \tau}$. Then we have

$$
Q^{n+1} \leq(1+\vartheta \tau) Q^{n} \leq \cdots \leq(1+\vartheta \tau)^{n+1} Q^{0} \leq \cdots \leq e^{\vartheta \tau(n+1)} Q^{0} .
$$

Because $Q^{0}=\frac{7}{9}\left\|I^{0}\right\|^{2}+\frac{2 h}{9} \sum_{j=1}^{J-1} I_{j}^{0} I_{j+1}^{0}+\left\|I_{x}^{0}\right\|^{2}+\left\|I_{x x}^{0}\right\|^{2}=0, Q^{n+1} \leq e^{\vartheta \tau(n+1)} Q^{0}=0$. We have

$$
Q^{n}=\frac{7}{9}\left\|I^{n}\right\|^{2}+\frac{2 h}{9} \sum_{j=1}^{J-1} I_{j}^{n} I_{j+1}^{n}+\left\|I_{x}^{n}\right\|^{2}+\left\|I_{x x}^{n}\right\|^{2}=0
$$


It is well known that

$$
\frac{5}{9}\left\|I^{n+1}\right\|^{2}+\left\|I_{x}^{n+1}\right\|^{2}+\left\|I_{x x}^{n+1}\right\|^{2} \leq Q^{n}=0 .
$$

So $\left\|I^{n+1}\right\|^{2}=\left\|U^{n+1}-V^{n+1}\right\|^{2}=0$. We can say that $U^{n+1}=V^{n+1}$.

\section{Some prior estimates for the difference solution}

In this section, we shall make some prior estimates for the numerical scheme.

Theorem 4.1 Assume that $u_{0} \in H_{0}^{2}\left[x_{l}, x_{r}\right]$ then there is the estimation for the solution of the numerical scheme (3.7)

$$
\left\|u_{x x}^{n}\right\| \leq C, \quad\left\|u_{x}^{n}\right\| \leq C, \quad\left\|u^{n}\right\|_{\infty} \leq C .
$$

Proof It follows from (3.12) that

$$
\frac{5}{9}\left\|u^{n}\right\|^{2}+\left(\frac{2}{9}\left\|u^{n}\right\|^{2}+\frac{2}{9} \sum_{i=1}^{J-1} u_{i}^{n} u_{i+1}^{n} h\right)+\left\|u_{x}^{n}\right\|^{2}+\left\|u_{x x}^{n}\right\|^{2}=C
$$

we obtain from (4.1)

$$
\frac{5}{9}\left\|u^{n}\right\|^{2}+\left\|u_{x}^{n}\right\|^{2}+\left\|u_{x x}^{n}\right\|^{2} \leq C .
$$

So

$$
\left\|u_{x x}^{n}\right\| \leq C, \quad\left\|u_{x}^{n}\right\| \leq C, \quad\left\|u^{n}\right\| \leq C .
$$

It follows from Lemma 2.4 that $\left\|u^{n}\right\|_{\infty} \leq C$.

Remark 4.1 Theorem 4.1 implies that the scheme (3.7)-(3.9) is unconditionally stable.

\section{Convergence and stability of the difference scheme}

Now, we consider the truncation error of scheme of (3.7). Firstly, we define the truncation error as follows:

$$
\begin{aligned}
E r_{j}^{n+\frac{1}{2}}= & \frac{1}{9 \tau}\left(v_{i-1}^{n+1}+7 v_{i}^{n+1}+v_{i+1}^{n+1}-v_{i-1}^{n}-7 v_{i}^{n}-v_{i+1}^{n}\right)+\left(v_{i}^{n+\frac{1}{2}}\right)_{\widehat{x}} \\
& -\left(v_{i}^{n+\frac{1}{2}}\right)_{x \widehat{x t}}+\left(v_{i}^{n+\frac{1}{2}}\right)_{x x \widehat{x x t}}+\frac{1}{3}\left(v_{i}^{n+\frac{1}{2}}\right)_{\widehat{x}}\left(v_{i-1}^{n+\frac{1}{2}}+v_{i}^{n+\frac{1}{2}}+v_{i+1}^{n+\frac{1}{2}}\right) .
\end{aligned}
$$

According to Taylor expansion and Lagrange interpolation, we obtain the following.

Lemma 5.1 Assume that $u_{0} \in H_{0}^{2}\left[x_{l}, x_{r}\right]$ and $u(x, t) \in C^{5,2}$, then the truncation errors of the numerical scheme (3.7) satisfy

$$
E r_{j}^{n+\frac{1}{2}}=O\left(\tau^{2}+h^{3}\right)
$$


Theorem 5.1 Assume that $u_{0} \in H_{0}^{2}\left[x_{l}, x_{r}\right]$ and $u(x, t) \in C^{5,2}$, then the solution of the $n u-$ merical scheme (3.7) converges to the solution of the initial-boundary value problem (1.5)(1.7) with order $O\left(\tau^{2}+h^{3}\right)$ by the $\|\cdot\|_{\infty}$ norm.

Proof Let $e_{j}^{n+\frac{1}{2}}=u\left(x_{j}, t^{n+\frac{1}{2}}\right)-u_{j}^{n+\frac{1}{2}}$. Subtracting (3.7) from (5.1), we have

$$
\begin{aligned}
\operatorname{Er}_{j}^{n+\frac{1}{2}}= & \frac{1}{9 \tau}\left(e_{j-1}^{n+1}+7 e_{j}^{n+1}+e_{j+1}^{n+1}-e_{j-1}^{n}-7 e_{j}^{n}-e_{j+1}^{n}\right)+\frac{1}{4}\left(\left(e_{j}^{n+1}\right)_{\widehat{x}}+\left(e_{j}^{n}\right)_{\widehat{x}}\right)-\left(e_{j}^{n+\frac{1}{2}}\right)_{x \widehat{x t}} \\
& +\left(e_{j}^{n+\frac{1}{2}}\right)_{x x \widehat{x x \hat{t}}}+\frac{1}{3}\left(u\left(x_{j}, t^{n+\frac{1}{2}}\right)\right)_{\widehat{x}}\left(u\left(x_{j-1}, t^{n+\frac{1}{2}}\right)+u\left(x_{j}, t^{n+\frac{1}{2}}\right)+u\left(x_{j+1}, t^{n+\frac{1}{2}}\right)\right) \\
& -\frac{1}{3}\left(u_{j}^{n+\frac{1}{2}}\right)_{\widehat{x}}\left(u_{j-1}^{n+\frac{1}{2}}+u_{j}^{n+\frac{1}{2}}+u_{j+1}^{n+\frac{1}{2}}\right) .
\end{aligned}
$$

For simple notation, the last two terms of (5.3) are defined by

$$
\begin{aligned}
Q= & \left(u\left(x_{j}, t^{n+\frac{1}{2}}\right)\right)_{\widehat{x}}\left(u\left(x_{j-1}, t^{n+\frac{1}{2}}\right)+u\left(x_{j}, t^{n+\frac{1}{2}}\right)+u\left(x_{j+1}, t^{n+\frac{1}{2}}\right)\right) \\
& -\frac{1}{3}\left(u_{j}^{n+\frac{1}{2}}\right)_{\widehat{x}}\left(u_{j-1}^{n+\frac{1}{2}}+u_{j}^{n+\frac{1}{2}}+u_{j+1}^{n+\frac{1}{2}}\right) .
\end{aligned}
$$

Computing the inner product of (5.3) with $2 e^{n+\frac{1}{2}}$ (i.e. $e^{n+1}+e^{n}$ ), we get

$$
\begin{aligned}
& \left(E r_{j}^{n+\frac{1}{2}}, 2 e^{n+\frac{1}{2}}\right) \\
& =\frac{7}{9 \tau}\left\|e^{n+1}\right\|^{2}+\frac{2 h}{9 \tau} \sum_{j=1}^{J-1} e_{j}^{n+1} e_{j+1}^{n+1}-\frac{7}{9 \tau}\left\|e^{n}\right\|^{2}-\frac{2 h}{9 \tau} \sum_{j=1}^{J-1} e_{j}^{n} e_{j+1}^{n}+\frac{1}{\tau}\left(\left\|e_{x}^{n+1}\right\|^{2}-\left\|e_{x}^{n}\right\|^{2}\right) \\
& \quad+\frac{1}{\tau}\left(\left\|e_{x x}^{n+1}\right\|^{2}-\left\|e_{x x}^{n}\right\|^{2}\right)+\left(Q, 2 e^{n+\frac{1}{2}}\right) .
\end{aligned}
$$

According to Theorem 3.3, we obtain

$$
-\left(Q, 2 e^{n+\frac{1}{2}}\right) \leq C\left(\left\|e^{n+1}\right\|^{2}+\left\|e^{n}\right\|^{2}+\left\|e_{x}^{n+1}\right\|^{2}+\left\|e_{x}^{n}\right\|^{2}\right) .
$$

In addition, obviously

$$
\left(E r_{j}^{n+\frac{1}{2}}, 2 e^{n+\frac{1}{2}}\right)=\left(E r_{j}^{n+\frac{1}{2}}, e^{n+1}+e^{n}\right) \leq\left\|E r^{n+\frac{1}{2}}\right\|^{2}+\frac{1}{2}\left(\left\|e^{n+1}\right\|^{2}+\left\|e^{n}\right\|^{2}\right) .
$$

Substituting (5.4) and (5.5) into (5.3), we have

$$
\begin{gathered}
\frac{7}{9}\left\|e^{n+1}\right\|^{2}+\frac{2 h}{9} \sum_{j=1}^{J-1} e_{j}^{n+1} e_{j+1}^{n+1}-\frac{7}{9}\left\|e^{n}\right\|^{2}-\frac{2 h}{9} \sum_{j=1}^{J-1} e_{j}^{n} e_{j+1}^{n} \\
+\left\|e_{x}^{n+1}\right\|^{2}-\left\|e_{x}^{n}\right\|^{2}+\left\|e_{x x}^{n+1}\right\|^{2}-\left\|e_{x x}^{n}\right\|^{2} \\
\leq \tau\left\|E r^{n+\frac{1}{2}}\right\|^{2}+\frac{\tau}{2}\left(\left\|e^{n+1}\right\|^{2}+\left\|e^{n}\right\|^{2}\right) \\
+C \tau\left(\left\|e^{n+1}\right\|^{2}+\left\|e^{n}\right\|^{2}+\left\|e_{x}^{n+1}\right\|^{2}+\left\|e_{x}^{n}\right\|^{2}\right) .
\end{gathered}
$$

Let $B^{n}=\frac{7}{9}\left\|e^{n}\right\|^{2}+\frac{2 h}{9} \sum_{j=1}^{J-1} e_{j}^{n} e_{j+1}^{n}+\left\|e_{x}^{n}\right\|^{2}+\left\|e_{x x}^{n}\right\|^{2}$, then (5.6) can be rewritten as

$$
B^{n+1}-B^{n} \leq \tau\left\|E r^{n+\frac{1}{2}}\right\|^{2}+C \tau\left(B^{n+1}+B^{n}\right) .
$$


So

$$
(1-C \tau)\left(B^{n+1}-B^{n}\right) \leq \tau\left\|E r^{n+\frac{1}{2}}\right\|^{2}+2 C \tau B^{n} .
$$

If $\tau$ is sufficiently small so that it satisfies $1-C \tau=\sigma>0$, then

$$
B^{n+1}-B^{n} \leq C \tau\left\|E r^{n+\frac{1}{2}}\right\|^{2}+C \tau B^{n} .
$$

Summing up (5.7) from 0 to $n-1$, we have

$$
B^{n}-B^{0} \leq C \tau \sum_{i=0}^{n-1}\left\|E r^{i+\frac{1}{2}}\right\|^{2}+C \tau \sum_{i=0}^{n-1} B^{i} .
$$

Noticing, $\tau \sum_{i=0}^{n-1}\left\|E r^{i+\frac{1}{2}}\right\|^{2} \leq n \tau \max _{0 \leq i \leq n-1}\left\|E r^{i+\frac{1}{2}}\right\|^{2} \leq T \cdot O\left(\tau^{2}+h^{2}\right)^{2}, e^{0}=0$. We get $B^{0}=0$.

Hence, from Lemma 2.5, we obtain

$$
B^{n} \leq O\left(\tau^{2}+h^{3}\right)^{2}
$$

That is,

$$
\frac{7}{9}\left\|e^{n}\right\|^{2}+\left\|e_{x}^{n}\right\|^{2}+\left\|e_{x x}^{n}\right\|^{2} \leq O\left(\tau^{2}+h^{3}\right)^{2} .
$$

So

$$
\left\|e^{n}\right\| \leq O\left(\tau^{2}+h^{3}\right), \quad\left\|e_{x}^{n}\right\| \leq O\left(\tau^{2}+h^{3}\right), \quad\left\|e_{x x}^{n}\right\| \leq O\left(\tau^{2}+h^{3}\right) .
$$

Using Lemma 2.4, we get $\left\|e^{n}\right\|_{\infty} \leq O\left(\tau^{2}+h^{3}\right)$.

Theorem 5.2 Under the conditions of Theorem 5.1, the solution of the numerical scheme (3.7) is unconditionally stable by the $\|\cdot\|_{\infty}$ norm.

\section{Numerical experiment}

In this section, we will calculate some numerical experiments to verify the correctness of our theoretical analysis in the above part.

Consider the following initial boundary value problem of the Rosenau-RLW equation:

$$
u_{t}-u_{x x t}+u_{x x x x t}+u_{x}+u u_{x}=0, \quad(x, t) \in(0,1) \times(0, T)
$$

with the initial condition

$$
u(x, 0)=x^{3}(1-x)^{3}, \quad x \in[0,1]
$$

and boundary conditions

$$
u(0, t)=u(1, t)=0, \quad u_{x x}(0, t)=u_{x x}(1, t)=0, \quad t \in[0, T] .
$$


Table 1 Absolute error of numerical solution at $\tau=0.02$

\begin{tabular}{lllll}
\hline$x$ & $J=10$ & $J=30$ & $J=50$ & $J=80$ \\
\hline 0.1 & $1.92 \mathrm{E}-08$ & $1.03 \mathrm{E}-09$ & $2.45 \mathrm{E}-10$ & $3.45 \mathrm{E}-12$ \\
0.2 & $3.23 \mathrm{E}-08$ & $1.63 \mathrm{E}-09$ & $3.47 \mathrm{E}-10$ & $5.25 \mathrm{E}-11$ \\
0.3 & $3.27 \mathrm{E}-08$ & $1.52 \mathrm{E}-09$ & $2.33 \mathrm{E}-10$ & $1.66 \mathrm{E}-10$ \\
0.4 & $2.09 \mathrm{E}-08$ & $7.95 \mathrm{E}-10$ & $5.49 \mathrm{E}-11$ & $3.21 \mathrm{E}-10$ \\
0.5 & $2.11 \mathrm{E}-09$ & $2.30 \mathrm{E}-10$ & $4.09 \mathrm{E}-10$ & $4.69 \mathrm{E}-10$ \\
0.6 & $1.68 \mathrm{E}-08$ & $1.23 \mathrm{E}-09$ & $7.20 \mathrm{E}-10$ & $5.70 \mathrm{E}-10$ \\
0.7 & $2.91 \mathrm{E}-08$ & $1.87 \mathrm{E}-09$ & $8.82 \mathrm{E}-10$ & $5.84 \mathrm{E}-10$ \\
0.8 & $2.96 \mathrm{E}-08$ & $1.86 \mathrm{E}-09$ & $8.07 \mathrm{E}-10$ & $4.85 \mathrm{E}-10$ \\
0.9 & $1.76 \mathrm{E}-08$ & $1.14 \mathrm{E}-09$ & $4.80 \mathrm{E}-10$ & $2.76 \mathrm{E}-10$ \\
\hline
\end{tabular}

Table 2 Discrete energy values at $h=\tau=0.02$

\begin{tabular}{llll}
\hline$t$ & $E^{n}$ & $E^{n}[21]$ & $E^{n}[20]$ \\
\hline 0.1 & 0.058374806402 & 0.058524796850 & 0.057831719901 \\
0.2 & 0.058374806389 & 0.058524796915 & 0.057831712420 \\
0.3 & 0.058374806373 & 0.058524796919 & 0.057831704931 \\
0.4 & 0.058374806345 & 0.058524796957 & 0.057831697437 \\
0.5 & 0.058374806324 & 0.058524796889 & 0.057831689928 \\
0.6 & 0.0583748062860 & 0.058524796925 & 0.057831682415 \\
0.7 & 0.058374806258 & 0.058524796877 & 0.057831674891 \\
0.8 & 0.058374806217 & 0.058524796939 & 0.057831667363 \\
0.9 & 0.058374806168 & 0.058524797018 & 0.057831659824 \\
1.0 & 0.058374806107 & 0.058524797061 & 0.057831652277 \\
\hline
\end{tabular}

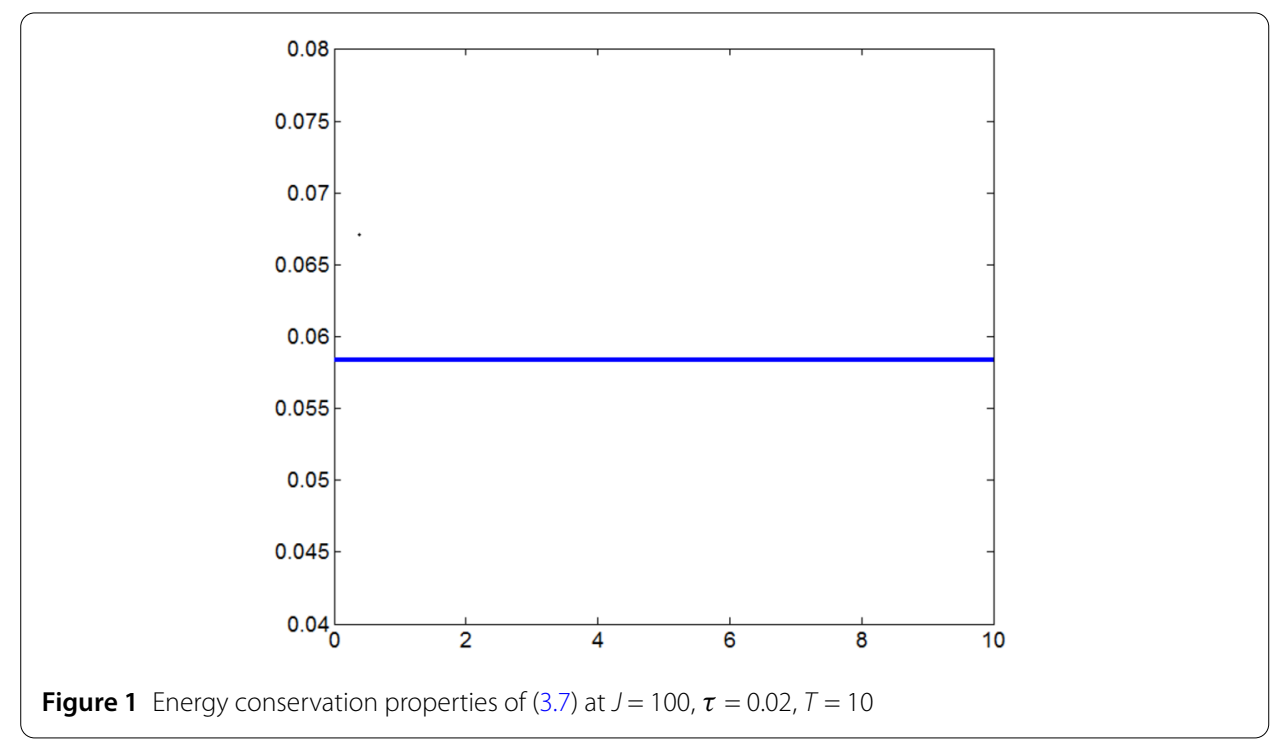

Because we do not know the exact solution of (6.1)-(6.3), we consider the numerical solution of fine grid, taking $h=\frac{1}{500}$, as the accurate solution of (6.1)-(6.3). Next we compare the numerical solution of the coarse grid and the numerical solution of fine grid. In order to obtain the error estimation, we consider the solution as a reference solution of the grid. In Table 1 , with the time step $\tau=0.02$, we give the absolute error between numerical solution and accurate solution under a different spatial step $h$.

According to the numerical results in Table 1, we can see that numerical format (3.7) is effective. 
In Table 2, with $h=\tau=0.01$, discrete energy values of numerical format (3.7) are given and are compared with the discrete energy values in the literature [20] and [21]. It can be seen that the multiple integral finite volume method preserves the conservation of discrete energy better than the numerical method in the literature [20] and [21]. Figure 1 shows the energy conservation properties of scheme (3.7) with $J=100, \tau=0.02, T=10$.

\title{
7 Conclusion
}

In this paper, we have presented a two-level implicit nonlinear numerical scheme for the Rosenau-RLW equation, which has a wide range of applications in physics. The uniqueness, convergence and stability with $O\left(\tau^{2}+h^{3}\right)$ of the numerical scheme were discussed in detail. The scheme kept the energy conservation characteristic of the original equation. Finally, a numerical experiment shows that our scheme is efficient.

\author{
Acknowledgements \\ The authors wish to thank the referees for their valuable suggestions. \\ Funding \\ This work is supported by National Natural Science Foundation of China (NO. 11526064), Fundamental Research Funds for \\ the Central Universities (NO. 3072019CF2403) and Graduate Education Fund (NO. 00211001060930).
}

Availability of data and materials

Data sharing not applicable to this article as no datasets were generated or analyzed during the current study.

Competing interests

The authors declare that they have no competing interests.

Authors' contributions

All authors contributed equally and significantly in writing this article. All authors read and approved the final manuscript.

Authors' information

The authors 1, 3 and 4 have been working at Harbin Engineering University for many years. The author Fang Li is a student. She has obtained her master degree of mathematics in Harbin Engineering University.

\section{Publisher's Note}

Springer Nature remains neutral with regard to jurisdictional claims in published maps and institutional affiliations.

Received: 11 January 2019 Accepted: 2 October 2019 Published online: 24 October 2019

\section{References}

1. Peregrine, D.H.: Calculations of the development of an undular bore. J. Fluid Mech. 25(2), 321-330 (1966)

2. Peregrine, D.H.: Long waves on a beach. J. Fluid Mech. 27(4), 815-827 (1967)

3. Rosenau, P.: A quasi-continuous description of a nonlinear transmission line. Phys. Scr. 34(6B), 827-829 (1986)

4. Rosenau, P.: Dynamics of dense discrete systems: high order effects. Prog. Theor. Phys. Suppl. 79(5), 1028-1042 (1988)

5. Park, M.A.: On the Rosenau equation. Mat. Apl. Comput. 9, 145-152 (1990)

6. Ghiloufi, A., Rahmeni, M., Omrani, K.: Convergence of two conservative high-order accurate difference schemes for the generalized Rosenau-Kawahara-RLW equation. Eng. Comput. 1-16 (2019)

7. Rouatbi, A., Achouri, T., Omrani, K.: High-order conservative difference scheme for a model of nonlinear dispersive equations. Comput. Appl. Math. 37(4), 4169-4195 (2018)

8. Ghiloufi, A., Omrani, K.: New conservative difference schemes with fourth-order accuracy for some model equation for nonlinear dispersive waves. Numer. Methods Partial Differ. Equ. 34(2), 451-500 (2017)

9. Ruoatbi, A., Rouis, M., Omrani, K.: Numerical scheme for a model of shallow water waves in $(2+1)$-dimensions. Comput. Math. Appl. 74(8), 1871-1884 (2017)

10. Atouani, N., Ouali, Y., Omrani, K.: Mixed finite element methods for the Rosenau equation. J. Appl. Math. Comput. $57(1-2), 393-420(2017)$

11. Atouani, N., Omrani, K.: On the convergence of conservative difference schemes for the $2 \mathrm{D}$ generalized Rosenau-Korteweg de Vries equation. Appl. Math. Comput. 250, 832-847 (2015)

12. Atouani, N., Omrani, K.: A new conservative high-order accurate difference scheme for the Rosenau equation. Appl. Anal. 11, 2435-2455 (2014)

13. Lu, J., Zhang, T.: Adaptive stabilized finite volume method and convergence analysis for the Oseen equations. Bound. Value Probl. 2018, 129 (2018)

14. Zhou, D., Mu, C.: Homogeneous initial-boundary value problem of the Rosenau equation posed on a finite interval. Appl. Math. Lett. 57, 7-12 (2016) 
15. Choo, S.M., Chung, S.K., Kim, K.I.: A discontinuous Galerkin method for the Rosenau equation. Appl. Numer. Math. 58(6), 783-799 (2008)

16. Omrani, K., Abidi, F., Achouri, T., et al.: A new conservative finite difference scheme for the Rosenau equation. Appl. Math. Comput. 201(1-2), 35-43 (2008)

17. Hu, J., Zheng, K.: Two conservative difference schemes for the generalized Rosenau equation. Bound. Value Probl. 2010(1), 500 (2010)

18. Wang, H., Wang, S.: Decay and scattering of small solutions for Rosenau equations. Appl. Math. Comput. 218(1), 115-123 (2011)

19. Wang, S., Su, X.: Global existence and asymptotic behavior of solution for Rosenau equation with Stokes damped term. Math. Methods Appl. Sci. 38(17), 3990-4000 (2015)

20. Zuo, J.M., Zhang, Y.M., Zhang, T.D., et al.: A new conservative difference scheme for the general Rosenau-RLW equation. Bound. Value Probl. 2010(1), 516 (2010)

21. Pan, X., Zhang, L.: On the convergence of a conservative numerical scheme for the usual Rosenau-RLW equation. Appl. Math. Model. 36(8), 3371-3378 (2012)

22. Atouani, N., Omrani, K.: Galerkin finite element method for the Rosenau-RLW equation. Comput. Math. Appl. 66(3), 289-303 (2013)

23. Thomée, V:: Galerkin Finite Element Methods for Parabolic Problems. Springer, Berlin (1984)

24. $\mathrm{Mu}, \mathrm{L}$., Wang, J.P., Ye, X:: A hybridized formulation for the weak Galerkin mixed finite element method. J. Comput. Appl. Math. 307, 335-345 (2016)

25. Wongsaijai, B., Poochinapan, K.: A three-level average implicit finite difference scheme to solve equation obtained by coupling the Rosenau-KdV equation and the Rosenau-RLW equation. Appl. Math. Comput. 245, 289-304 (2014)

26. Yagmurlu, N.M., Karaagac, B., Kutluay, S.: Numerical solutions of Rosenau-RLW equation using Galerkin cubic B-spline finite element method. Am. J. Comput. Appl. Math. 7(1), 1-10 (2017)

27. Pan, X., Zheng, K., Zhang, L.: Finite difference discretization of the Rosenau-RLW equation. Appl. Anal. 92(12), 2578-2589 (2013)

28. Wang, H., Li, S., Wang, J.: A conservative weighted finite difference scheme for the generalized Rosenau-RLW equation. Appl. Comput. Math.-Bak. 36(1), 63-78 (2017)

29. Browder, F.E.: Existence and uniqueness theorems for solutions of nonlinear boundary value problems. Proc. Symp. Appl. Math. 17, 24-49 (1965)

30. Zhou, Y: Applications of Discrete Functional Analysis to the Finite Difference Method. Pergamon, Elmsford (1991)

\section{Submit your manuscript to a SpringerOpen ${ }^{\circ}$ journal and benefit from:}

- Convenient online submission

- Rigorous peer review

- Open access: articles freely available online

- High visibility within the field

- Retaining the copyright to your article

Submit your next manuscript at $\boldsymbol{~ s p r i n g e r o p e n . c o m ~}$ 